(C) 2019. This manuscript version is made available under the CC-BY-NC-ND 4.0 license http://creativecommons.org/licenses/by-nc-nd/4.0/

\title{
Routes for enhanced magnetism in Ni-Mn-In metamagnetic shape memory alloys
}

\author{
J. López-García,a,b V. Sánchez-Alarcos ${ }^{b, c}$, V. Recarte ${ }^{b, c}$, J. I. Pérez-Landazábal ${ }^{b, c}$, O. Fabelo ${ }^{a}$, E. \\ Cesarid, and J. A. Rodríguez-Velamazán ${ }^{a *}$ \\ a Institut Laue Langevin, 71, Avenue des Martyrs, 38042 Grenoble Cedex, France \\ ${ }^{\text {b }}$ Departamento Física. Universidad Pública de Navarra, Campus de Arrosadia, 31006 Pamplona, Spain \\ 'Institute for Advanced Materials (INAMAT), Universidad Pública de Navarra, Campus de Arrosadía, 31006 \\ Pamplona, Spain \\ ${ }^{\mathrm{d}}$ Departament de Física. Universitat de les Illes Balears. Ctra. De Valldemossa, km 7.5, E-07122 Palma de \\ Mallorca, Spain
}

*Corresponding author. E-mail address: velamazan@ill.eu

\begin{abstract}
We provide in-depth physical insight into the enhancement of the magnetic properties of metamagnetic shape memory alloys produced by thermal treatment and cobalt doping. We use neutron scattering to study the atomic order and magnetic structures in the austenitic phases of $\mathrm{Ni}_{50} \mathrm{Mn}_{34} \mid \mathrm{n}_{16}$ and $\mathrm{Ni}_{45} \mathrm{CO}_{5} \mathrm{Mn}_{37} \mathrm{In}_{13}$ alloys in two different states induced by thermal treatments. The increase of the magnetization in the austenite phase, particularly by cobalt doping, is explained by the enhanced ferromagnetic coupling between the magnetic moments located in octahedral sites. The spin density maps obtained from polarized neutron diffraction reveal the magnetic interaction pathways responsible for this coupling scheme.
\end{abstract}

Keywords: Ferromagnetic shape memory alloys; Neutron diffraction; Magnetic properties; Magnetic coupling

The vast interest and the widespread research effort dedicated to magnetic shape memory alloys (SMAs) in the last two decades stems from their potential as multifunctional materials, based on the interplay between a first-order structural transition - the martensitic transformation (MT) - and the magnetic ordering. ${ }^{1}$ Within these alloys, the so-called metamagnetic ones - $\mathrm{Ni}_{2} \mathrm{MnX}(\mathrm{X}=\mathrm{In}, \mathrm{Sn}, \mathrm{Sb})-$ present a particularly large variation in the magnetization $(\Delta M)$ associated to the $M T$, which allows inducing the reverse MT with moderate magnetic fields.[1-4] Their applicability in areas like magnetic refrigeration - based on magnetocaloric effect [5-7] - or in sensing - based on giant magnetoresistance $[8,9]-$ is therefore significantly improved. It appears straightforward that the control of the magnetostructural properties and the comprehensive understanding of the physics of the system is crucial to develop these materials. In this work, we focus in the magnetic coupling between magnetic atoms in the different crystallographic sites, and how this coupling can be modified to produce improved magnetic properties.

The MT in magnetic SMAs takes place between a high-symmetry cubic $(F m \overline{3} m)$ ferromagnetic austenitic phase and a magnetic martensitic phase with lower symmetry, giving different structures depending of the composition.[10-14] The magnetic moments are mainly confined to the Mn atoms, which are not nearest neighbors, the indirect exchange interactions being strongly dependent on the 
interatomic distances. $[15,16]$ In ordered stoichiometric $\mathrm{Ni}_{50} \mathrm{Mn}_{25} \mathrm{X}_{25}$ all the $\mathrm{Mn}$ atoms locate in the $4 a$ sites of the austenite, with the moments aligned parallel. In off-stoichiometry compositions and/or disordered structures, the ferromagnetic coupling is in principle reduced by the presence of $\mathrm{Mn}$ moments at $4 b$ sites that tend to couple antiferromagnetically.

Composition and atomic order are therefore key parameters that govern the magnetostructural properties of these systems. In particular, Co doping in Ni-Mn-In and Ni-Mn-Sn metamagnetic SMAs has been shown to increase both the Curie temperature $\left(T_{C}\right)$ and the spontaneous magnetization of the austenite by the enhancement of the ferromagnetic coupling, $[17,18]$ while hindering the ferromagnetic ordering in martensite, thus leading to an increase of the change $\Delta M$ at the $M T$ and consequently improving the magnetic properties relevant for applications.[2,19-21] On the other hand, the Curie and MT temperatures, as well as the spontaneous magnetization, are particularly sensitive to the long-range atomic order,[22-25] thermal treatments being one of the most direct ways to tune such a parameter.

We have selected the $\mathrm{Ni}_{50} \mathrm{Mn}_{34} \mid \mathrm{n}_{16}$ and $\mathrm{Ni}_{45} \mathrm{Co}_{5} \mathrm{Mn}_{37} \mathrm{n}_{13}$ alloys in two different states induced by thermal treatments (water quenching, WQ, and slow cooling, SC) to study in detail the effect of cobalt doping and atomic order in the magnetic properties of these metamagnetic SMAs in terms of the magnetic coupling between the different magnetic moments. These alloys have been chosen by balancing compositional similarity, transforming character (occurrence of MT), and available temperature range in both paramagnetic and ferromagnetic austenitic phases. Besides, both specific compositions have been extensively studied in the literature. Following the characterization of the macroscopic magnetic behavior by magnetometry measurements, we have employed neutron powder diffraction and single-crystal polarized neutron diffraction [26] to study in detail the atomic order and magnetic structures in the austenitic phases of the alloys.

The ternary $\mathrm{Ni}_{50} \mathrm{Mn}_{34} \mid \mathrm{n}_{16}$ and quaternary $\mathrm{Ni}_{45} \mathrm{CO}_{5} \mathrm{Mn}_{37} \mathrm{In}_{13}$ alloys were synthetized from high purity elements by arc-melting under protective argon atmosphere. The obtained bulks were homogenized at $1173 \mathrm{~K}$ during $24 \mathrm{~h}$ and quenched in ice water or slow cooled in air in order to induce different atomic order degrees. A single crystal of slow-cooled $\mathrm{Ni}_{45} \mathrm{C}_{5} \mathrm{Mn}_{37} \mathrm{In}_{13}$ alloy, grown by the Bridgman method and cut into a small cube of $3 \times 3 \times 3 \mathrm{~mm}$ was used for the single-crystal neutron diffraction experiments. The composition of the elaborated alloys was analyzed by EDS in a Jeol JSM-5610LV Scanning Electron Microscope (SEM), giving $\mathrm{Ni}_{50} \mathrm{Mn}_{34} \mathrm{n}_{16}$ and $\mathrm{Ni}_{45} \mathrm{Co}_{5} \mathrm{Mn}_{37} \mathrm{In}_{13}$ for the ternary and quaternary powder samples, respectively, and $\mathrm{Ni}_{45.5} \mathrm{CO}_{5.5} \mathrm{Mn}_{36.5} \mathrm{In}_{12.4}$ for the single crystal sample. The macroscopic magnetic characterization of the alloys was performed using a Quantum Design MPMS XL-7 SQUID magnetometer using powder samples. Additionally, magnetization measurements were performed on a single crystal of the slow-cooled $\mathrm{Ni}_{45} \mathrm{CO}_{5} \mathrm{Mn}_{37} \mathrm{In}_{13}$ alloy oriented with the magnetic field parallel to the $c$-axis. Neutron diffraction experiments were performed in D1B $(\lambda=1.28 \AA \AA$ and $2.52 \AA)$ and D2B $(\lambda=1.59 \AA ̊)$, powder diffractometers, $D 9(\lambda=0.8 \AA$ and $0.5 \AA)$ single-crystal diffractometer and D3 $(\lambda=0.8 \AA \AA)$ polarized-neutron diffractometer at Institut Laue-Langevin in Grenoble. The polarized neutron diffraction measurements were carried out in paramagnetic austenite phase, with an applied magnetic field along the $c$-axis. The data treatment was carried out using the programs of the Fullprof Suite.[27] The maximum entropy method,[28] as implemented in Dysnomia program, [29] was used to obtain spin density maps from the polarized neutron diffraction data.

Figure 1 shows the temperature dependence of the magnetization. On cooling from ca. $380 \mathrm{~K}$, a second order transition takes place, characterized by a significant increase of the magnetization of the alloy and indicating the onset of the magnetic ordering $\left(T_{C}\right)$. On further cooling, an abrupt decrease in the magnetization reveals the occurrence of the MT, the first order character of this transition being confirmed by the hysteresis observed on warming. Finally, at lower temperatures, changes in the 
magnetic signal indicate different magnetic transitions depending on the composition and order degree. The comparison of the curves of the four different samples (Fig. 1) reveals the effects of thermal treatment and Co doping. Regarding the transition temperatures (Table 1), Co-doping produces substantial increments in both the $\mathrm{T}_{\mathrm{c}}$ and the MT. On the other hand, the thermal treatment produces a marked displacement of the MT to lower temperatures and an increase of the $T_{C}$ when comparing SC with WQ samples. Concerning $\Delta M$, Co-doping gives the most pronounced effect at low fields, due to the substantial increase produced in the magnetization of the austenite phase (Fig. 1). If we consider the saturation magnetization in this phase $(M(H)$ curves in Fig. 1, inset), for powder samples, atomic ordering (slow cooling treatment) and Co-doping are shown to produce a similar increase of ca. $35 \%$ with respect to the ternary WQ alloy, with the increment reaching ca. $60 \%$ by the combination of both in the SC quaternary alloy. This behavior is in concordance with previous works. $[18,19,24,30]$ The saturation magnetization obtained for a single crystal of the SC quaternary alloy oriented with the applied magnetic field parallel to the easy magnetization axis is even $10 \%$ higher than for a powder sample.

The atomic order and the magnetic coupling in the austenitic phase of the studied alloys have been analyzed by unpolarized neutron diffraction. The results, in the form of occupancies of the different crystallographic sites and magnetic moments per site, are summarized in Table 1. The diffraction data obtained correspond to the Heusler $L 2_{1}$ structure with $F m \overline{3} m$ space group. The occupancies have been determined in the paramagnetic phase, assuming as starting point an ordered system. Then, using soft restraints according to the composition of the alloys to reduce the number of free parameters and taking advantage of the negative scattering factor of $\mathrm{Mn}$, the different species were allowed in all crystallographic sites. Since for some of the species the refined occupancy recurrently gave negative or close-to-zero values, these were fixed to zero. In the case of the quaternary SC alloy, powder and single crystal data have been combined, improving the reliability of the results. The refined occupancies show how the atomic order increases in SC alloys with respect to WQ ones. In the SC samples, a maximum atomic order is obtained, with the $\mathrm{Mn}$ atoms almost fully occupying the $4 a$ sites (only a small quantity of ca. $2 \%$ of $\mathrm{In}$ is refined in this positon) and practically all the available $\mathrm{In}$ and $\mathrm{Ni}$ atoms located respectively in the $4 b$ and $8 c$ sites. The $\mathrm{Mn}$ atoms in excess with respect to the stoichiometric composition complete the occupancy of the $4 b$ site, while in the quaternary alloy the Co atoms complete the occupancy of the $8 c$ site, which is not fully occupied by Ni since the composition of this alloy is defective in Ni with respect to the stoichiometric one. The atomic disorder in the ternary quenched alloy is driven by $\mathrm{Ni}$ atoms that are present in all sites, letting space for $\ln$ in $8 c$ site, with some $\mathrm{Mn}$ migrating from $4 a$ to $4 b$ position to complete the occupancy of this last one. In the quaternary WQ alloy, Mn fully occupies the $4 a$ sites and the excess of this species locates in the $4 b$ site. In turn, a certain amount of In and, to a lesser extent, $\mathrm{Co}$, move from its corresponding site, being both present in the $4 b$ and $8 c$ sites.

The measurements in the ferromagnetic austenite phase in both SC and WQ conditions were performed at similar reduced temperatures $\left(T / T_{c} \approx 0.8\right.$ ) to allow comparison (ca. $245 \mathrm{~K}$ and $310 \mathrm{~K}$ for the ternary and quaternary alloys, respectively). The low temperature patterns show an increase of the intensity of some nuclear peaks (namely the $\left(\begin{array}{lll}2 & 0 & 0\end{array}\right)$ and the $\left(\begin{array}{lll}1 & 1 & 1\end{array}\right)$ reflections) due to a magnetic ordering with a propagation vector $\boldsymbol{k}=0$. This magnetic intensity (see insets in figure 2 ) gives a first indication about the magnetic coupling, since the magnetic intensity ratio between the $(200)$ and the (1 11 1) reflections should increase as the coupling between the atoms in $4 a$ and $4 b$ sites becomes more ferromagnetic. In line with the magnetometry results, Co-doping produces a noticeable increase of the ferromagnetic coupling. In order to avoid correlations of the magnetic structure analysis with other parameters, we have carefully extracted the magnetic intensity by subtraction of the data taken in the paramagnetic phase. The magnetic integrated intensities have been used to obtain the magnetic moments by the simulated annealing method, $[31,32]$ and then these values have been applied in the 
Rietveld fit of the diffraction patterns of the ferromagnetic phase, together with the site occupancies determined in the paramagnetic phase. In order to reduce the number of free parameters, the magnetic moments in the $8 \mathrm{c}$ site have been fixed to the values corresponding to $\mathrm{Ni}$ and $\mathrm{Co}$ atoms carrying a moment of 0.2 and $1.0 \mu_{B}$ respectively, in accordance with theoretical calculations [33] and with previous neutron diffraction studies.[18]

The analysis agrees with most of the magnetic moment being carried by $\mathrm{Mn}$ atoms, which locate only in the octahedral $4 a$ and $4 b$ sites (table 1), with values approaching to $2 \mu_{B}$ and $1 \mu_{B}$ in the $4 a$ and $4 b$ sites, respectively (similar results were reported by Brown et al. for the ternary $\mathrm{Ni}_{46} \mathrm{Mn}_{41} \mathrm{In}_{13}$ alloy)[12]. Although the absolute values of the magnetic moments can be affected by the weak magnetic signal compared to the total intensity and the large error bars, several conclusions may be drawn by the relative values. In the ternary alloys, the resultant overall coupling between magnetic moments in the $4 a$ and $4 b$ positions is ferromagnetic, but with a relatively lower value of the magnetic moment in the $4 b$ position. When the atomic disorder and the defects are reduced by slow cooling thermal treatment in the SC alloy, the total magnetic moment increases, with a marked increase in the $4 a$ site and the coupling of the moment in the $4 b$ becoming slightly more ferromagnetic. The reduced presence of $\mathrm{Mn}$ moments at $4 b$ sites, which tend to couple antiferromagnetically,[18] appears as a possible cause for the enhancement of the ferromagnetic coupling. Additionally, the thermal relaxation reduces the strain and the antiphase boundaries in the sample and, as a consequence, favors the ferromagnetic moments.[34] Yet the most pronounced effect in the coupling between $4 a$ and $4 b$ sites is that of Codoping, with values of the magnetic moment in the $4 b$ sites markedly higher despite the higher amount of $\mathrm{Mn}$ atoms in these sites with respect to the ternary alloy. In a similar way, in the NiMnSn system,[18] it has been observed that Co-doping produces a substantial increase of the ferromagnetic coupling, involving even a switch of the coupling between sites $4 a$ and $4 b$ from antiferromagnetic to ferromagnetic. Finally, both effects add up in the ordered quaternary alloy when Co-doping is combined with the slow cooling thermal treatment, resulting in enhanced magnetic moments with stronger ferromagnetic coupling and giving a total increase of the total ordered magnetic moment reaching ca. $60 \%$ with respect to the ternary disordered alloy (similarly to the observed in the macroscopic magnetization).

In order to gain more insight into the magnetic coupling and the interaction pathways in this system, we have performed polarized neutron diffraction measurements on a single-crystal of the atomically ordered $\mathrm{Ni}_{45} \mathrm{CO}_{5} \mathrm{Mn}_{37} \mathrm{In}_{13}$ alloy. The obtained spin density maps (Fig. 3) show the magnetic moments in all sites polarized parallel, and therefore coupled ferromagnetically, in agreement with the unpolarized neutron diffraction results above. From the spin-density between sites, one can deduce that the stronger interaction occurs between $4 a$ and $4 b$ sites (see Fig.3), corresponding to a distance of $a / 2$ in the $L 2_{1}$ cubic structure, followed in intensity by the interaction between $4 a-4 a$ or $4 b-4 b$ sites, corresponding to a distance of $a / \sqrt{ } 2$. According to these maps, the interaction of $8 c$ with the $4 a$ and $4 b$ ones (distance $a \sqrt{ } 3 / 4$ ) should be very weak, but of the utmost importance since the replacement of $\mathrm{Ni}$ by Co, occurring mainly in the $8 c$ position, has been seen to produce a significant increase in the ferromagnetic coupling. The well-known Bethe-Slater curve, that qualitatively describes the variation in the intensity of the direct exchange coupling as a function of the ratio of the interatomic distance to the radius of the $3 \mathrm{~d}$ electron shell,[35] can help understanding this behavior. When Co replaces $\mathrm{Ni}$ at a given crystallographic position, the strength of the ferromagnetic interaction is increased due to its bigger $3 \mathrm{~d}$ electron shell radius. Also, DFT studies indicate that the ferromagnetic exchange interactions become significantly stronger when Co occupies the 8c sites instead of Ni.[33] Consequently, the introduction of $\mathrm{Co}$, that mainly replaces $\mathrm{Ni}$ in $8 c$ positions, enhances the overall ferromagnetic interactions,[17] and since the moments in $8 c$ positions tend to couple ferromagnetically with its 
nearest neighbors in $4 a$ and $4 b$ sites, which in turn are coupled by strong interactions, this favors the ferromagnetic coupling of the Mn moments.

In conclusion, the enhancement of the magnetic properties of metamagnetic Ni-Mn-In-based shape memory alloys through thermal treatments and cobalt doping has been studied at the macroscopic and microscopic levels by means of magnetometry and neutron diffraction measurements, respectively. Magnetometry reveals an increase of both the magnetic ordering temperature and of the variation in the magnetization associated to the martensitic transformation, which implies an improvement of the magnetic properties relevant for applications. Neutron diffraction has been used to investigate the origin of these effects by analyzing the atomic order and magnetic arrangement in the austenite phase of $\mathrm{Ni}_{50} \mathrm{Mn}_{34} \mathrm{In}_{16}$ and $\mathrm{Ni}_{45} \mathrm{CO}_{5} \mathrm{Mn}_{37} \mathrm{In}_{13}$ in two different atomic order states induced by thermal treatments. The higher degree of atomic order, together with the reduction of strains and defects induced by slow cooling thermal treatment produces an increase in the total ordered magnetic moment and a slight enhancement of the ferromagnetic coupling between the magnetic moments located in octahedral sites, while cobalt doping has a stronger effect in increasing the ferromagnetic coupling, which explains the noticeable effect in the magnetization. The spin density maps obtained from polarized neutron diffraction reveal the magnetic interactions responsible for this coupling scheme. Despite the weak interaction of the moments in $8 c$ sites with the rest of the magnetic moments, $\mathrm{Co}$, that mainly replaces $\mathrm{Ni}$ in $8 \mathrm{c}$ positions, tends to couple ferromagnetically with its nearest neighbors in $4 a$ and $4 b$ sites (where the magnetic atoms are mainly $\mathrm{Mn}$ ), which in turn are coupled by strong interactions, favoring the overall ferromagnetic coupling.

Acknowledgments: This work has been carried out with the financial support of the Spanish "Ministerio de Ciencia, Innovación y Universidades" (Project numbers MAT2015-65165-C2-R and MAT201456116-C4-1-R). ILL and SpINS are acknowledgment for the beam time allocation: experiments 5-41497, EASY-379, 5-31-2484 (https://doi.org/10.5291/ILL-DATA.5-31-2484) and CRG-1905. J. LópezGacía acknowledges ILL for Ph.D. contract.

\section{References.}

[1] M. Acet, L. Manosa, and A. Planes, Handbook of Magnetic Materials edited by, K. H. J. Buschow, Elsevier, Amsterdam, 2011, Vol. 19, p. 231

[2] R. Kainuma, Y. Imano, W. Ito, Y. Sutou, H. Morito, S. Okamoto, K. Kitakami, A. Oikawa, O. Fujita, T. Kanomata, K. Ishida, Nature 439 (2006) 957.

[3] K. Koyama, K. Watanabe, T. Kanomata, R. Kainuma, K. Oikawa, K. Ishida, Appl. Phys. Lett. 88 (2006) 132505.

[4] K. Oikawa, W. Ito, Y. Imano, Y. Sutou, R. Kainuma, K. Ishida, S. Okamoto, O. Kitakami, T. Kanomata, Appl. Phys. Lett. 88 (2006) 122507

[5] A. Planes, LI. Mañosa, M. Acet, J. Phys. Condens. Matter 21 (2009) 233201

[6] J. Liu, T. Gottschall, K.P. Skokov, J.K. Moore, O. Gutfleisch, Nature Mater. 11 (2012) 620

[7] J. I. Perez-Landazabal, V. Recarte, V. Sánchez-Alarcos, J.J. Beato-López, J.A. Rodríguez-Velamazán, J.

Sánchez-Marcos, C. Gómez-Polo, E. Cesari, Sci. Rep., 7 (2017) 13328

[8] S. Pandey, A. Quetz, A. Aryal, I. Dubenko, D. Mazumdar, S. Stadler, N. Ali, Magnetochemistry 3 (2017) 3

[9] Z.B. Li, W. Hu, F.H. Chen, M.G. Zhang, Z.Z. Li, B. Yang, X. Zhao, L. Zuo, J. Magn. Magn. Mater., 452 (2018) 249

[10] P. J. Brown, J. Crangle, T. Kanomata, M. Matsumoto, K.-U. Neumann, B. Ouladdiaf and K. R. A. Ziebeck, J. Phys. Condens. Matter, 14, (2002) 10159

[11] P. J. Brown, A.P. Gandy, K. Ishida, R. Kainuma, T. Kanomata, K.-U. Neumann, K. Oikawa, B. Ouladdiaf and K.R.A Ziebeck, J. Phys. Condens. Matter, 18 (2006) 2249 
[12] P. J. Brown, A.P. Gandy, R. Kainuma, T. Kanomata, K.-U. Neumann, K. Oikawa, B. Ouladdiaf, A. Sheikh and K.R.A Ziebeck, J. Phys. Condens. Matter, 23 (2011) 456004

[13] L. Righi, F. Albertini, L. Pareti, A. Paoluzi, and G. Calestani, Acta Mater., 55 (2007) 5237

[14] S. Singh, V. Petricek, P. Rajput, Adrian H. Hill, E. Suard, S. R. Barman and D. Pandey, Phys. Rev. B, 90 (2014) 14109

[15] E. Sasioglu, L.M. Sandratskii, P. Bruno, Phys. Rev. B 70 (2004) 024427

[16] E. Sasioglu, L.M. Sandratskii, P. Bruno, Phys. Rev. B 77 (2008) 064417

[17] K. Ollefs, Ch. Schöppner, I. Titov, R. Meckenstock, F. Wilhelm, A. Rogalev, J. Liu, O. Gutfleisch, M. Farle, H. Wende, and M. Acet, Phys Rev B 92 (2015) 224429

[18] R.Y. Umetsu, A. Sheikh, W. Ito, B. Ouladdiaf, K.R.A. Ziebeck, T. Kanomata, R.Kainuma, Appl Phys Lett 98 (2011) 042507

[19] R. Kainuma, Y. Imano, W. Ito, H. Morito, Y. Sutou, K. Oikawa, A. Fujita, K. Ishida, S. Okamoto, O. Kitakami, and T. Kanomata, Appl Phys Lett. 88 (2006) 192513

[20] V. Recarte, J.I. Pérez-Landazábal, E. Cesari,S. Kustov, J. Appl. Physics 107 (2010) 053501

[21] L. Huang, D.Y. Cong, H.L. Suo, Y.D. Wang, Appl. Phys. Lett. 104 (2014) 132407

[22] V. Sánchez-Alarcos, V. Recarte, J. I. Pérez-Landazábal, and G. J. Cuello, Acta Mater., 55 (2007) 3883

[23] W. Ito, M. Nagasako, R. Y. Umetsu, R. Kainuma, T. Kanomata, and K. Ishida, Appl. Phys. Lett., 93 (2008) 232503

[24] V. Recarte, J. I. Pérez-Landazábal, and V. Sánchez-Alarcos, J. Alloys Compd., 536 (2012) S308-S311

[25] S. Kustov, M. L. Corro, J. Pons, E. Cesari, Appl Phys Lett 94 (2009) 191901

[26] P. Lázpita, J. M. Barandiarán, J. Gutiérrez, C. Mondelli, A. Sozinov, and V. A. Chernenko, Phys. Rev. Lett., 119 (2017) 155701,

[27] J. Rodríguez-Carvajal, Phys. B Condens. Matter, 192 (1993) 55

[28] R. J. Papoular and B. Gillon, EPL Europhys. Lett., 13 (1990) 429

[29] K. Momma, T. Ikeda, A.A. Belik and F. Izumi, Powder Diffraction, 28 (2013) 184

[30] S. Singh, I. Glavatskyy, and C. Biswas, J. Alloys Compd., 601(2014) 108

[31] S. Kirpatrick, C. Gelatt, M. Vecci, Science, 220 (1983) 671.

[32] J. Rodriguez-Carvajal, Mater. Sci. Forum 268 (2001) 378

[33] Y. Kurtulus, R. Dronskowski, G. D. Samolyuk, and V. P. Antropov, Phys.Rev. B 71, (2005) 014425

[34] I. Unzueta, J. López-García, V. Sánchez-Alarcos, V. Recarte, J. I. Pérez-Landazábal, J. A. RodríguezVelamazán, J. S. Garitaonandia, J. A. García, and F. Plazaola, Appl. Phys. Lett., 110 (2017) 181908

[35] S. Chikazumi, Physics of Ferromagnetism, Oxford University Press, New York, 1997, p. 125, ISBN 0-19851776-9

[36] V. Sánchez-Alarcos, V. Recarte, J. I. Pérez-Landazábal, E. Cesari, J.A. Rodríguez-Velamazán, Entropy 16 (2014) 2756 
Table 1. Transition temperatures of the studied alloys. Nuclear and magnetic structure parameters obtained by unpolarized neutron diffraction: occupancy and magnetic moment determined for $4 a, 4 b$ and $8 c$ sites. Calculated total magnetic moment obtained from neutron diffraction $\left(m_{t o t}\right)$ and macroscopic magnetization at 60kOe $\left(m_{60 \mathrm{kO}}\right)$ measured in the ferromagnetic austenitic phase.

\begin{tabular}{|c|c|c|c|c|c|c|c|c|c|c|}
\hline \multirow[b]{3}{*}{ Alloy } & \multirow{3}{*}{$\begin{array}{l}\text { MT } \\
\text { (K) }\end{array}$} & \multirow{3}{*}{$\begin{array}{l}\mathrm{T}_{\mathrm{C}} \\
(\mathrm{K})\end{array}$} & \multicolumn{6}{|c|}{ Sites } & \multirow{2}{*}{\multicolumn{2}{|c|}{$\begin{array}{c}\text { Magnetic } \\
\text { moment } \\
\left(\mu_{B} / f . u .\right)\end{array}$}} \\
\hline & & & \multicolumn{2}{|c|}{$4 a$} & \multicolumn{2}{|c|}{$4 b$} & \multicolumn{2}{|l|}{$8 c$} & & \\
\hline & & & Occ & $\mu_{B}$ & Occ & $\mu_{B}$ & Occ & $\mu_{B}^{*}$ & $m_{\text {tot }}$ & $m_{60 \mathrm{kO}}$ \\
\hline $\begin{array}{l}\text { Ni-Mn-In } \\
W Q\end{array}$ & 227 & 298 & $\begin{array}{c}0.94(1) \mathrm{Mn} \\
0.06(1) \mathrm{Ni}\end{array}$ & $1.76(10)$ & $\begin{array}{c}0.41(2) \mathrm{Mn} \\
0.47(2) \mathrm{In} \\
0.09(1) \mathrm{Ni} \\
\end{array}$ & $0.65(15)$ & $\begin{array}{l}0.88(5) \mathrm{Ni} \\
0.12(5) \mathrm{In}\end{array}$ & 0.176 & $2.76(25)$ & 2.78 \\
\hline $\begin{array}{l}\text { Ni-Mn-In } \\
\text { SC }\end{array}$ & 190 & 309 & $\begin{array}{c}0.98(2) \mathrm{Mn} \\
0.02(2) \mathrm{In}\end{array}$ & $2.18(17)$ & $\begin{array}{l}0.38(1) \mathrm{Mn} \\
0.62(1) \mathrm{In}\end{array}$ & $0.71(16)$ & $1.00(5) \mathrm{Ni}$ & 0.200 & $3.29(33)$ & 3.74 \\
\hline $\begin{array}{l}\text { Ni-Co-Mn- } \\
\text { In WQ }\end{array}$ & 305 & 360 & $1.00(2) \mathrm{Mn}$ & $1.63(13)$ & $\begin{array}{c}0.51(1) \mathrm{Mn} \\
0.47(1) \mathrm{In} \\
0.02(2) \mathrm{Co}\end{array}$ & $1.16(23)$ & \begin{tabular}{|l}
$0.88(2) \mathrm{Ni}$ \\
$0.09(6) \mathrm{Co}$ \\
$0.03(4) \mathrm{In}$
\end{tabular} & 0.268 & $3.33(36)$ & 3.70 \\
\hline $\begin{array}{l}\text { Ni-Co-Mn- } \\
\text { In SC }\end{array}$ & 232 & $\begin{aligned} \sim & 395 \\
& {[36] }\end{aligned}$ & $\begin{array}{c}0.969(5) \mathrm{Mn} \\
0.020(6) \mathrm{In}\end{array}$ & $2.06(12)$ & $\begin{array}{c}0.484(5) \mathrm{Mn} \\
0.503(4) \mathrm{In}\end{array}$ & $1.20(19)$ & $\begin{array}{l}0.898(8) \mathrm{Ni} \\
0.094(8) \mathrm{Co}\end{array}$ & 0.274 & $3.81(31)$ & 4.44 \\
\hline
\end{tabular}

*Magnetic moments for $\mathrm{Ni}$ and $\mathrm{Co}$ atoms fixed at 0.2 and $1.0 \mu_{B}$ respectively. 


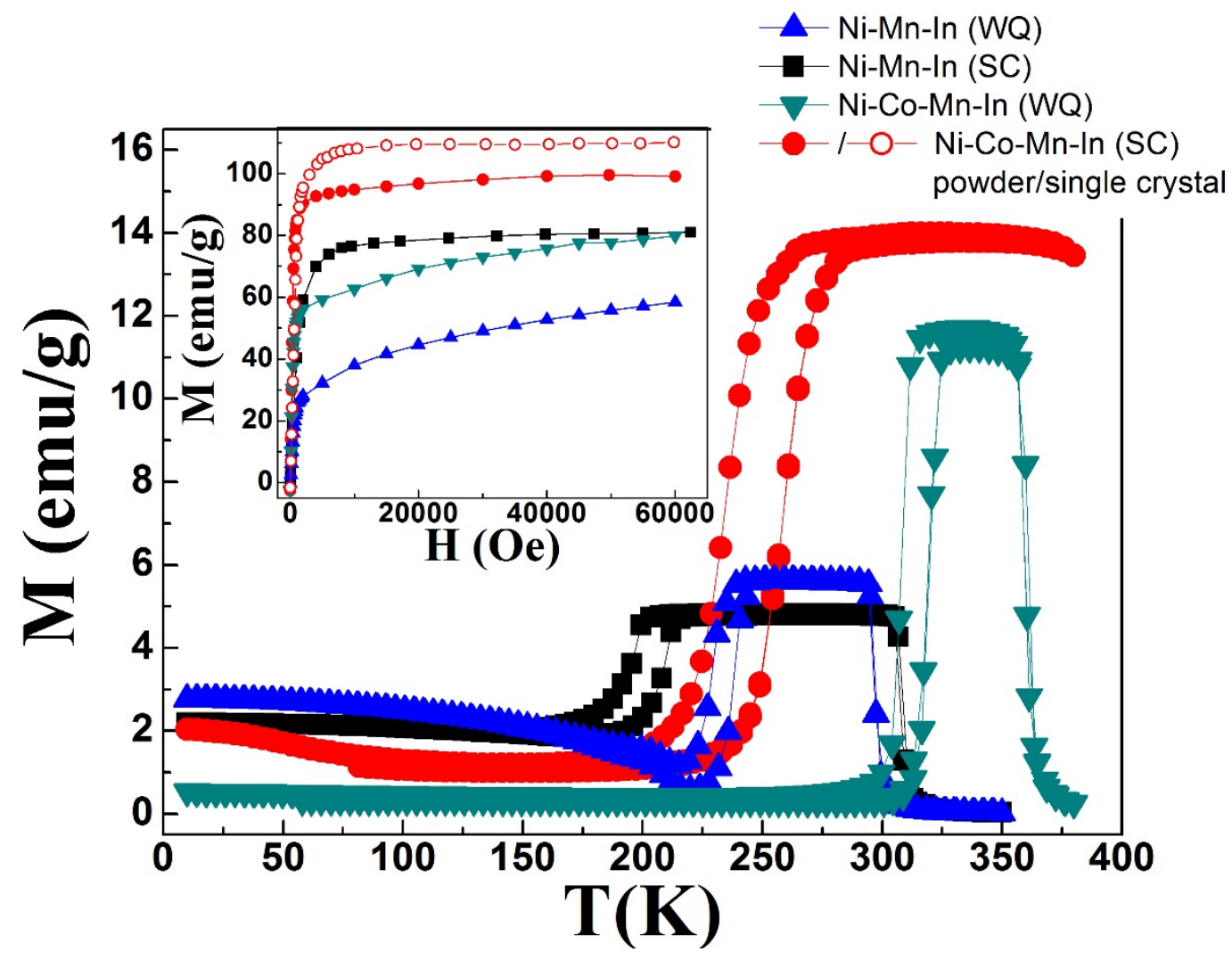

Fig. 1. Thermomagnetization curves at 100 Oe applied magnetic field. Inset: Magnetization dependence with applied magnetic field in austenitic ferromagnetic state (ca. $265 \mathrm{~K}$ and $310 \mathrm{~K}$ for the ternary and quaternary alloys, respectively). 


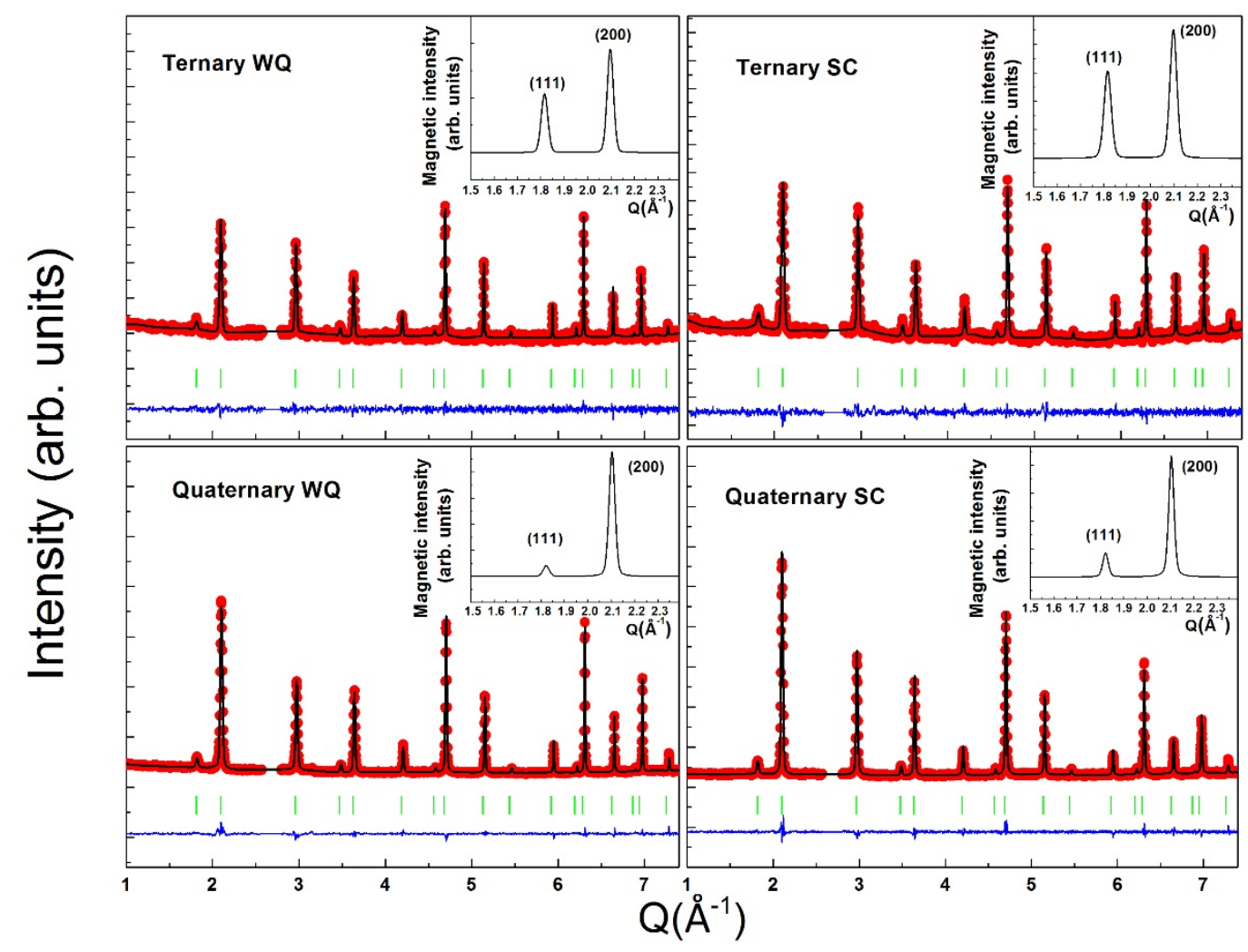

Fig. 2. Powder neutron diffraction patterns of $\mathrm{Ni}_{50} \mathrm{Mn}_{34} \mathrm{In}_{16}$ and $\mathrm{Ni}_{45} \mathrm{Co}_{5} \mathrm{Mn}_{37} \mathrm{In}_{13}$ in the different thermal treatment states measured in ferromagnetic austenitic phase. Red symbols represent the experimental data (contamination from aluminum of the sample environment around $2.7 \AA^{-1}$ has been removed from the diffractograms). Black and blue lines represent the observed, calculated and difference patterns, respectively; the green marks indicate the Bragg reflections. Insets: Calculated magnetic intensity contribution for the $\left(\begin{array}{lll}1 & 1 & 1\end{array}\right)$ and $\left(\begin{array}{lll}2 & 0 & 0\end{array}\right)$ reflections. 


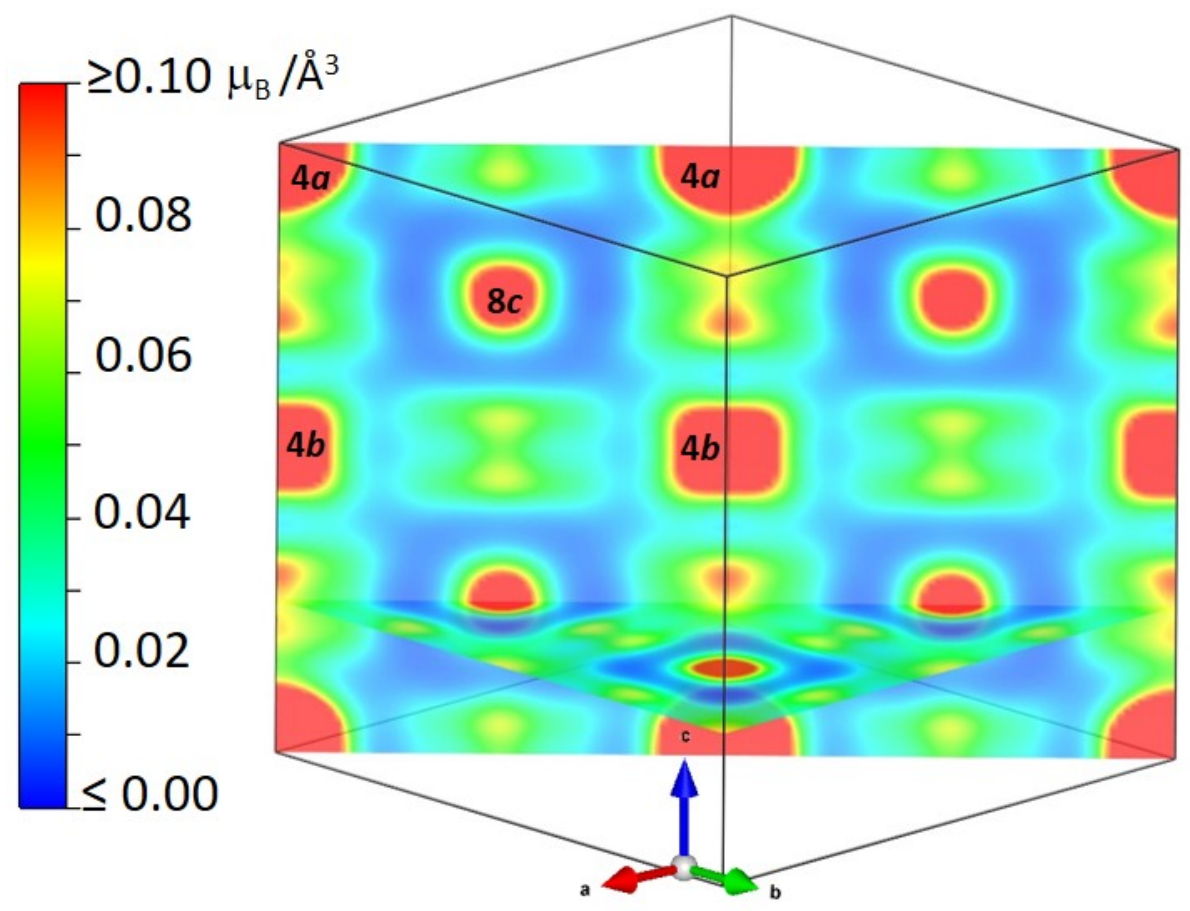

Fig. 3. Spin density obtained by maximum entropy method in (110) and (001) planes for $\mathrm{Ni}_{45} \mathrm{CO}_{5} \mathrm{Mn}_{37} \mathrm{In} \mathrm{n}_{13}$ single crystal measured in paramagnetic austenite phase $(450 \mathrm{~K})$, with an applied magnetic field of 8 Tesla along the $c$-axis. 